Research Article

\title{
Nonlinear Numerical Simulation for Oscillating Pressure in Cavity and Passive Control Research
}

\author{
Binqi Chen $(\mathbb{D})$ and Yiding Wang $(1)$ \\ School of Aeronautics and Astronautics, University of Electronic Science and Technology of China, Chengdu, China \\ Correspondence should be addressed to Yiding Wang; wyding127@163.com
}

Received 31 July 2019; Revised 8 November 2019; Accepted 25 November 2019; Published 20 December 2019

Academic Editor: Marco Pizzarelli

Copyright (c) 2019 Binqi Chen and Yiding Wang. This is an open access article distributed under the Creative Commons Attribution License, which permits unrestricted use, distribution, and reproduction in any medium, provided the original work is properly cited.

\begin{abstract}
Prediction of oscillating pressure is a key technology for cavity research. Nonlinear Acoustic Solver (NLAS) is applied to analyze aeroacoustics recently. A nonlinear numerical solver is combined with the Reynolds-averaged Navier-Stokes (RANS) method. RANS is applied to solving the flow field around a cavity, and average solution of initial turbulent statistics is obtained which contains the basic characteristics of the average flow field and statistic description of turbulence fluctuation. The source of acoustic generation is reconstructed, and the spreading of oscillating pressure is simulated precisely. According to the comparison of the cavity noise calculation and experimental results under Mach numbers $0.6,0.85$, and 1.35, it indicates that NLAS is capable to predict oscillating pressure of cavity flow from subsonic to supersonic with acceptable deviation. On this basis, the contribution to oscillating pressure suppression made by passive control such as rectangular fence (RF) and square tooth spoiler (STS) is investigated. It is found that these two passive control methods can lessen the total noise.
\end{abstract}

\section{Introduction}

Cavity structure is particularly common in the field of aeronautics and astronautics, such as under the carriage wheel well and weapon bay. For combat aircrafts, when the weapon bay doors are opened and exposed to the outflow, turbulence characteristics of the flow field would induce cavity flow instability. The weapon bay undertakes great aeroacoustic noise load, which leads to fatigue damage of the internal weapon and aircraft structure and also affects the surrounding aircraft systems. In addition, the instable cavity flow produces additional moments for the internal weapon, which adds restrictions on the operating envelope of the internal weapon bay.

In recent years, computational aeroacoustics (CAA) have been a trend to predict the acoustic noise phenomena due to high cost in the wind tunnel experiments. Gloerfelt et al. [1] investigate the acoustic field of a cavity using the direct numerical simulation (DNS). Theoretically, direct numerical simulation (DNS) can be a helpful tool since it can resolve all scales of the turbulent flow field. But the application of DNS is still restricted to low Reynolds numbers considering the available computational resources $[2,3]$. Unsteady Reynolds-averaged Navier-Stokes (URANS) [4-6] is effective to study the cavity flows. However, while coupled with aeroacoustic analysis, simulations [7-9] show that URANS is insufficient for predicting the spectral broadband of the acoustic spectrum, since the turbulent energy is lost in the time-averaging progress. Large eddy simulation (LES) becomes popular for predicting flow and noise [10, 11]. It is still computationally too expensive especially for high Reynolds number flow. Recent effort is made by a hybrid RANS/LES method known as Detached Eddy Simulation (DES) which is a compromising method between URANS and DNS. It is proposed by Spalart et al. [12] and widely applied [13-16]. Batten et al. [17] point out that these hybrid methods remain expensive and uncertain because the current methods largely rely on the dissipation 
of the subgrid scale model, and a substantial difficulty exists in transferring statistical turbulence energy data between RANS and LES regions [18].

Nonlinear Acoustic Solver (NLAS) combined the advantages between conventional acoustic analogy and hybrid RANS/LES methods together. It was proposed by Batten et al. in 2002 [19] which could consider subgrid scale sound sources by reconstruction of turbulence physical quantities. It reduces the mesh resolution without loss of precision. The NLAS method has been applied for predicting noise of jet and automotive field $[20,21]$ which proves that it is a promising research method.

Although some progress on the numerical method of cavity flow has been made, there are still few applications of the NLAS method on cavity problems. In this paper, application of the NLAS method on cavity noise and passive control is investigated. A cavity with a length to depth ratio of 5:1 is studied, and local Reynolds stress tensor and heat flux are obtained by calculating RANS equations using cubic a $k-\varepsilon$ model and then interpolated into acoustic mesh. Turbulence is reconstructed based on the statistic average result. Reliability of the NLAS method on predicting the cavity noise is validated by comparing the simulation result with the test result.

A lot of research on cavity flow control has been undertaken, and in these literatures, flow control methods can be mainly classified: passive control, active open-loop control, and active close-loop control [22, 23]. For the passive control, there is only simple geometry modification without any input of outer energy. Passive control is more cost-effective than active control, which achieves the goal of reducing noise by destructing the Rossiter feedback mechanism [24]. However, the best effect of passive control is obtained only when the aircraft is under the designed Mach number. Results [25-29] demonstrated that the size of the leading-edge control device has a significant impact on the noise control effect and also find that the cavity flow type will vary along with the variety of control measures such as SW, STS, trailing edge ramp (TR), and FTS. In this paper, the NLAS method is applied into research on the suppression effect of the passive control method for cavity noise.

\section{Numerical Simulation Methodology}

Nonlinear Acoustic Solver (NLAS) is a low-diffusion solver which simulates aerodynamic noise generation and propagation from a turbulent flow which is statistically steady. This numerical acoustic solver can take the data from a relatively simple RANS model instead of a LES model. In the initial step, the flow is described by a baseline of the mean flow and statistical superimposed turbulent fluctuations. Then, the NLAS generates acoustic sources from the given set of statistics using a reconstruction procedure. Finally, a high-resolution solver is used to simulate the resulting propagation of the oscillating pressure. The NLAS method can propagate the resolved scales of pressure disturbances on the original (RANS) mesh or on a separated acoustic mesh by interpolation of the acoustic output data. NLAS accounts for noise generation from structures at the subgrid scale relying on the previous mean flow where the perturbations are computed using statistical models. The NLAS solver considers quantities in the Navier-Stokes equations divided into mean and fluctuating parts: $\phi=\bar{\phi}+\phi^{\prime}$. A system of perturbation equations is performed by replacing the fluctuation and mean equations in the Navier-Stokes equations, which are referred to as nonlinear disturbance equations (NLDE):

$$
\frac{\partial q^{\prime}}{\partial t}+\frac{\partial F_{i}^{\prime}}{\partial x_{i}}-\frac{\partial\left(F_{i}^{v}\right)^{\prime}}{\partial x_{i}}=-\frac{\partial \bar{q}}{\partial t}-\frac{\partial \bar{F}_{i}}{\partial x_{i}}+\frac{\partial \overline{F_{i}^{v}}}{\partial x_{i}}
$$

where $q^{\prime}$ is the fluctuation term, $\bar{q}$ is the transient average term, $F_{i}^{\prime}$ is the nonviscous fluctuation term, $\bar{F}_{i}$ is the nonviscous average term, $\left(F_{i}^{v}\right)^{\prime}$ is the viscous fluctuation term, and $\bar{F}_{i}^{v}$ is the viscous average term. These terms can be solved as

$$
\begin{aligned}
& q^{\prime}=\left[\begin{array}{c}
\rho^{\prime} \\
\bar{\rho} u_{j}^{\prime}+\rho^{\prime} \bar{u}_{j}+\rho^{\prime} u_{j}^{\prime} \\
e^{\prime}
\end{array}\right], \\
& \left(F_{i}^{v}\right)=\left[\begin{array}{c}
0 \\
\tau^{\prime}{ }_{i j} \\
-\theta^{\prime}{ }_{i}+u^{\prime}{ }_{k} \bar{\tau}_{k i}+\bar{u}_{k} \tau^{\prime}{ }_{k i}
\end{array}\right] \text {, } \\
& \begin{aligned}
& F_{i}^{\prime}= {\left[\begin{array}{c}
\bar{\rho} u_{i}^{\prime}+\rho^{\prime} \bar{u}_{i} \\
\rho^{\prime} \bar{u}_{i} \bar{u}_{j}+\bar{\rho} \bar{u}_{i} u_{j}^{\prime}+\bar{\rho} u_{i}^{\prime} \bar{u}_{j}+p^{\prime} \delta_{i j} \\
u_{i}^{\prime}(\bar{e}+\bar{p})+\bar{u}_{i}\left(e^{\prime}+p^{\prime}\right)
\end{array}\right] } \\
& \rho^{\prime} u_{i}^{\prime} \\
&+\left[\begin{array}{c}
\bar{\rho} u_{i}^{\prime} u_{j}^{\prime}+\rho^{\prime} u_{i}^{\prime} \bar{u}_{j}+\rho^{\prime} \bar{u}_{i} u_{j}^{\prime}+\rho^{\prime} u_{i}^{\prime} u_{j}^{\prime} \\
u_{i}^{\prime}\left(e^{\prime}+p^{\prime}\right)
\end{array}\right],
\end{aligned}
\end{aligned}
$$

$$
\begin{gathered}
\bar{q}=\left[\begin{array}{c}
\bar{\rho} \\
\bar{\rho} \bar{u}_{j} \\
\bar{e}
\end{array}\right], \\
\bar{F}_{i}=\left[\begin{array}{c}
\bar{\rho}^{\prime} \bar{u}_{i} \\
\bar{\rho} \bar{u}_{i} \bar{u}_{j}+\bar{p} \delta_{i j} \\
\bar{u}_{i}(\bar{e}+\bar{p})
\end{array}\right], \\
\bar{F}_{i}^{v}=\left[\begin{array}{c}
0 \\
\tau^{\prime}{ }_{i j} \\
-\bar{\theta}_{i}+\bar{u}_{k} \bar{\tau}_{k i}
\end{array}\right] .
\end{gathered}
$$


In the above, values of $i, j, k$ are $1,2,3$ and represent three coordinate orientations, respectively; $\rho$ is density of flow; $p$ represents pressure; $u_{i}$ represents velocity of $x, y, z$ coordinate orientation; $x_{i}$ is the distance of three coordinate orientations; $\bar{e}$ and $e^{\prime}$ are the mean term and fluctuation term of unit volume, respectively; $\delta_{i j}$ is Kronecker function; $\tau^{\prime}{ }_{i j}$ is the fluctuation term of shear stress, and $\theta^{\prime}$ is the fluctuation term of heat transfer. Neglect density fluctuations and take time averages of the above equations:

$$
\begin{aligned}
\overline{L H S} & =\overline{R H S}=\frac{\partial R_{i}}{\partial x_{i}}, \\
R_{i} & =\left[\begin{array}{c}
\frac{0}{\bar{\rho}} \overline{u_{i}^{\prime} u_{j}^{\prime}} \\
\overline{c_{p}} \overline{\rho T^{\prime} u_{i}^{\prime}}+\bar{\rho} \overline{u_{i}^{\prime} u^{\prime}} \bar{u}_{k}+\frac{1}{2} \bar{\rho} \overline{u_{k}^{\prime} u_{k}^{\prime} u_{i}^{\prime}}+\overline{u_{k}^{\prime} \tau_{k i}}
\end{array}\right],
\end{aligned}
$$

where $R_{i}$ is related to the standard Reynolds stress tensor and turbulent heat flux, $C_{p}$ is the pressure coefficient, and $T^{\prime}$ is the fluctuation term of temperature. These unknown terms are the key of acoustic solution. Unsolved small-scale variables can be obtained by reconstructing the turbulence statistical results from RANS solving and used to produce subgrid source terms. The method of turbulence reconstruction proposed by Batten is as

$$
\begin{aligned}
u_{i}\left(x_{j}, t\right)= & a_{i k} \sqrt{\frac{2}{N}} \sum_{n=1}^{N}\left[p_{k}^{n} \cos \left(\widehat{d}_{j}^{n} \widehat{x}_{j}+w^{n} \widehat{t}\right)\right. \\
& \left.+q_{k}^{n} \sin \left(\hat{d}_{j}^{n} \widehat{x}_{j}+w^{n} \widehat{t}\right)\right]
\end{aligned}
$$

where

$$
\begin{aligned}
\widehat{x}_{j} & =\frac{2 \pi x_{j}}{l}, \\
\widehat{t} & =\frac{2 \pi t}{\tau}, \\
\widehat{d}_{j}^{n} & =d_{j}^{n} \frac{l}{\tau c^{n}}, \\
c^{n} & =\sqrt{\frac{3}{2} \overline{u^{\prime}{ }_{l}^{\prime}{ }_{m}^{\prime}} \frac{d_{l}^{n} d_{m}^{n}}{d_{k}^{n} d_{k}^{n}},} \\
p_{i}^{n} & =\varepsilon_{i j k} \eta_{j}^{n} d_{k}^{n}, \\
q_{i}^{n} & =\varepsilon_{i j k} \xi_{j}^{n} d_{k}^{n}, \\
\eta_{i}^{n}, \xi_{i}^{n} & \sim N(0,1), \\
\omega^{n} & \sim N(1,1), \quad d_{i}^{n} \sim N(0,1 / 2) .
\end{aligned}
$$

In the above expressions, $l$ is the turbulence scale, $\tau$ is the time scale, $\varepsilon_{i j k}$ is the permutation tensor of vector product operation, $N(\alpha, \beta)$ denotes a Gaussian normal variable with a mean of $\alpha$ and standard deviation $\beta ; a_{i j}$ is the Cholesky decomposition of local Reynolds stress tensor. For positive definite Reynolds stress tensor $\overline{u_{i}, u_{j}}, a_{i j}$ can be obtained:

$$
a_{i j}=\left[\begin{array}{ccc}
\frac{\sqrt{\overline{u_{1}^{\prime} u_{1}^{\prime}}}}{\overline{u_{1}^{\prime} u_{2}^{\prime}}} & 0 & 0 \\
\frac{a_{11}}{\overline{u_{2}^{\prime} u_{2}^{\prime}}-a_{21}^{2}} & 0 \\
\overline{\frac{u_{1}^{\prime} u_{3}^{\prime}}{a_{11}}} & \frac{\overline{u_{2}^{\prime} u_{3}^{\prime}}-a_{21} a_{31}}{a_{22}} & \sqrt{\overline{u_{3}^{\prime} u_{3}^{\prime}}-a_{31}^{2}-a_{32}^{2}}
\end{array}\right] .
$$

\section{Validation of NLAS Method}

3.1. Validation Model. M219 cavity is a representative model for cavity acoustic analysis, and a series of tests are carried out on M219 under QinetiQ wind tunnel shown as Figure 1 [30]. The configuration consisted of a rectangular cavity cut into a flat plate of length $1828.8 \mathrm{~mm}$ and width $431.8 \mathrm{~mm}$. The leading edge of the cavity was located $787.4 \mathrm{~mm}$ downstream from the plate leading edge with a length $508 \mathrm{~mm}$. The M219 cavity has an aspect ratio of $L: D: W=5: 1: 1$ with 10 equally spaced Kulite pressure sensors on the ceiling to measure the time histories of pressure with a sample frequency of $6 \mathrm{kHz}$.

From Figure 2, it could be seen that the computational domain of RANS which is consistent with M219 cavity is composed of four structural blocks. Flow direction $(X)$ is from $-8 \mathrm{D}$ to $12 \mathrm{D}$, spanwise direction is from $-2 \mathrm{D}$ to $2 \mathrm{D}$, and the surface normal is from $-1 \mathrm{D}$ to $4 \mathrm{D}$. To simulate the statistical average results of turbulent fluctuation, a nonlinear anisotropic turbulent model is used for RANS calculation. The cubic $k$-epsilon mode is adopted in this paper because it considers the influence of different positions to the eddy-viscosity coefficient by matrix approximation that is consistent with the physical aspects better. The height of the first mesh layer from the wall is $5 \times 10^{-2} \mathrm{~mm}$ with 2600 cells in total. The Reynolds number based on cavity depth and free-stream velocity is $7 \times 10^{6}$. For supersonic flow calculated by RANS, all condition-prescribed boundaries were applied on the inflow with Mach number $M=1.35$, pressure $P=62064 \mathrm{~Pa}$, and temperature $T=263 \mathrm{~K}$. A characteristicbased boundary was applied on the far field, and the physical quantities on outflow were extrapolated from the inner grid. For subsonic flow calculated by RANS, characteristic-based boundary conditions were applied on the inflow, outflow, and upper boundaries.

Separate computing mesh is used for acoustic calculation. The surface boundary is a nonslip adiabatic wall; solving precision in the surface domain is guaranteed by the wall function method. Local Reynolds stress tensor and statistical average of heat flux calculated by RANS are interpolated into acoustics mesh; then, according to the statistical average 

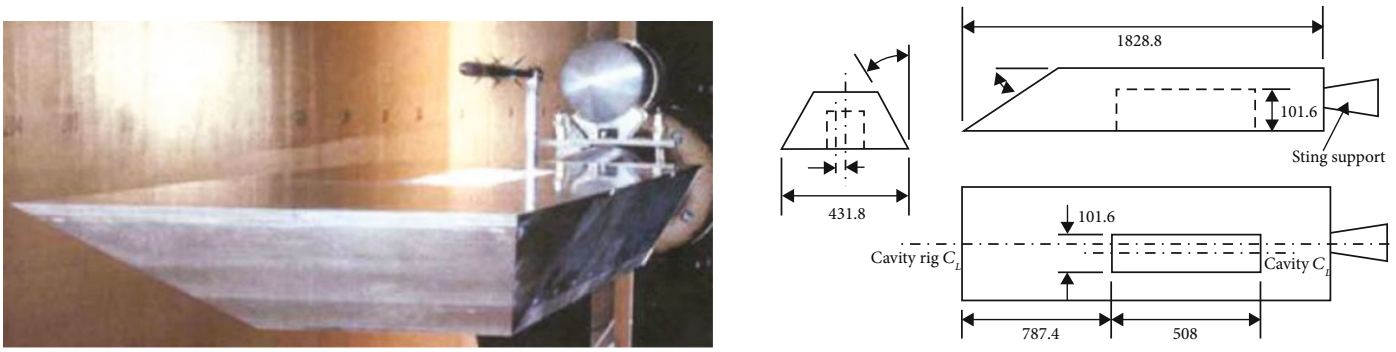

Figure 1: Noise test of M219 cavity in wind tunnel.

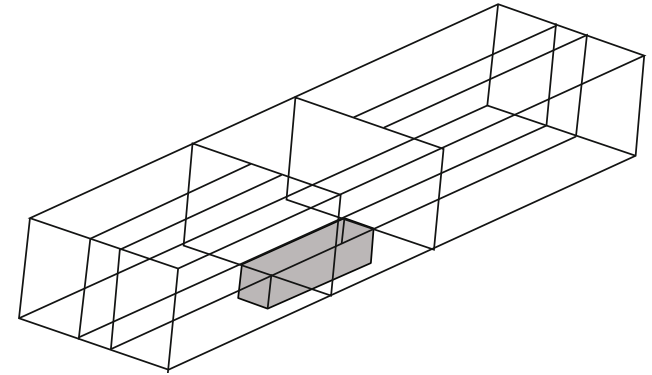

Figure 2: Computational domain of RANS.

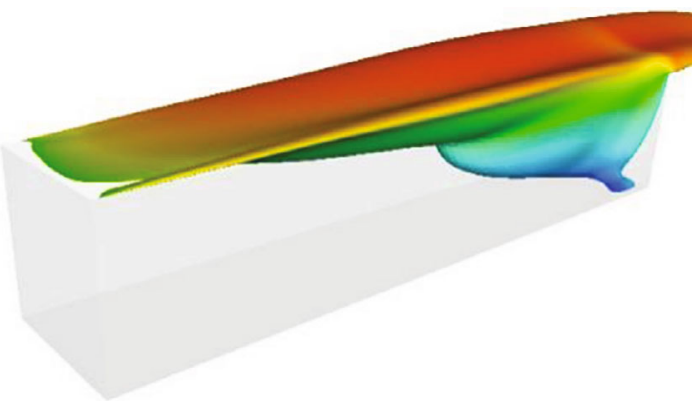

Figure 3: Isosurface of $10 \% k_{\max }$ calculated by RANS (shaded with streamwise velocity).

results, a synthetic reconstruction of turbulence is carried out. A computational domain contains surrounding areas of the acoustic source. Figure 3 is $10 \%$ isosurface of maximum turbulent kinetic energy $k_{\max }$ calculated by RANS. In this domain which is the main area of the acoustic source, turbulent fluctuation is the most violent. Therefore, the mesh in this domain is of great importance for acoustic calculation. A new boundary condition should be set as the absorbed layer; data of the far field and attenuation layer are provided by RANS.

Since the requirement of mesh is not strict and the computational domain is reduced, the acoustic mesh is much more coarse with 1200 cells in total which is reduced significantly compared with RANS mesh. Comparison of RANS and acoustics meshes is shown in Figure 4.

The benefits of this are that the acoustic solver can operate on more isotropic cells (particularly in the near-wall region, where a grid converged RANS solution is already available), resulting in a reduction in the overall number of mesh points from the relaxed near-wall requirements and a suitably truncated outer domain. Truncated outer boundaries in NLAS are assigned self-tuning absorbing layer boundary conditions, with far field (and damping layer) data provided by the (a priori) RANS solution. This provides a good description of the outer boundaries and minimizes spurious wave reflections back into the simulation domain, even for boundaries located close to the source region of interest. Compared with direct numerical simulation (DNS), the reduced grid requirements of a traditional LES are rather minimal, particularly in the near-wall region. Hybrid RANS/LES methods can achieve a reduction in mesh size by eliminating the mesh requirements in planes parallel to the wall (the normal-to-wall resolution is still required for the near-wall RANS modeling). NLAS further relaxes these meshing requirements, since a priori RANS statistics are always available, even on coarser regions of the NLAS mesh.

A governing equation of RANS is solved by the finite volume method; nonviscous terms are dispersed by secondorder TVD format. Viscous terms are dispersed by central difference format; an implicit algorithm is adopted for time marching. Calculating space and time discretization format of NLAS is the same as RANS. Time step size $\Delta t$ is $2 \times 10^{-5}$ s, and the total calculation step is 20000 .

Free-stream Mach numbers are consistent with the M219 wind tunnel test, by which numbers are $0.6,0.85$, and 1.35 and can verify the effectiveness and correctness of the cavity noise simulation using the NLAS method under subsonic, transonic, and supersonic flow conditions. Ten points are used to record the pressure variations under the bottom center line of cavity and indicated by K20 K29, shown as Figure 5.

3.2. Result Analysis. For the cavity above, when the incoming flow reaches the leading edge, the flow will cross the cavity without touching the bottom of the cavity. The shear layer over the cavity will become unstable and impact on the rear wall. The pressure in the rear part of the cavity rises, which will induce the appearance of shock wave under the condition of supersonic flow. The complex flow environment inside the cavity can lead to fluctuation in the rear part of the cavity and then generate noise. Noise spreads via circulating air to the leading edge, which contributes to the separation of the shear layer. When at a certain phase condition, an acoustic feedback loop is formed and selfsustained oscillation inside the cavity occurs. 


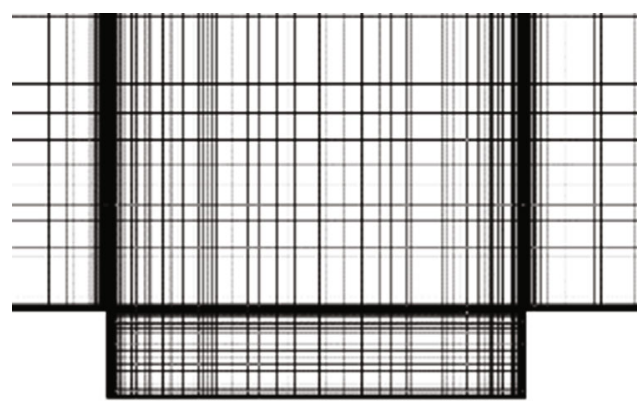

(a) RANS mesh

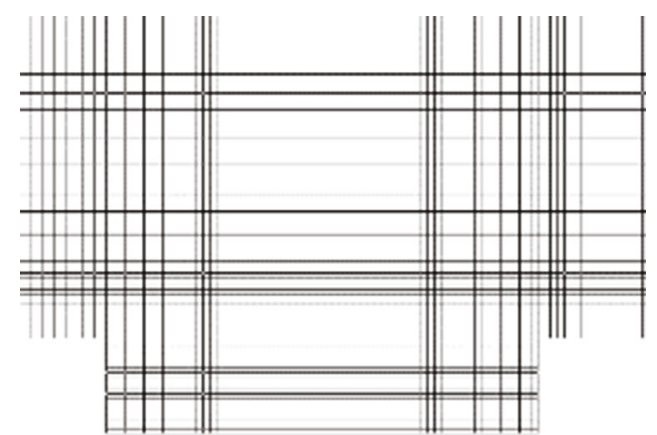

(b) Acoustic mesh

FIgURE 4: Comparison of RANS and acoustic meshes.

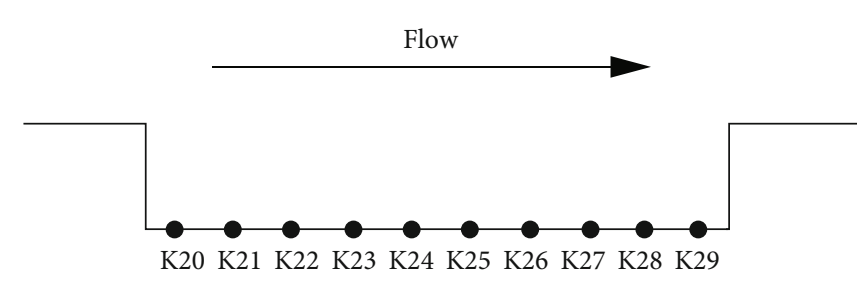

FIGURE 5: Monitoring locations of oscillating pressure.

Figures 6-8 show the sound pressure level results at three testing points located along the central line of cavity bottom $X / L=0.25,0.55$, and 0.95 , respectively, when $\mathrm{Ma}=0.6,0.85$, and 1.35. Sound pressure level spectrum curves of the three testing points are compared with experimental results. Oscillating pressure does not have periodicity during the initial time of the calculation. However, after the initial stage, oscillating pressure presents a certain periodicity and remains, which fits the oscillating pressure data that are needed and also the corresponding data of noise spreading. In order to avoid the irregular oscillating pressure during initial time affecting the solution of the noise, the initial stage is not considered and only the oscillating data during $0.1 \mathrm{~s} \sim 0.4 \mathrm{~s}$ are adopted in this paper.

From the calculation results, it could be seen that different positions under different Mach numbers have the consistent modal. That satisfies well with the typical frequency spectrum from the literature [30]. Calculating aerodynamic noise by this method, the spectrum modals of the previous 4 orders can be captured. What is more, besides slight difference in several modals, all the domain frequencies can be captured accurately. This illustrates the high accuracy of this method. When $\mathrm{Ma}=1.35$, the distribution of the sound pressure level at the measuring noise points concentrates during $130 \sim 170 \mathrm{~dB}$. The upstream sound pressure level is a little low while the downstream of that is a little high. For the interior weapon cabin having the similar structure, such high sound pressure level can contribute to fatigue damage of the cabin and weapons inside the cabin, and this kind of distribution will generate a nose up pitching moment on the weapon inside the cabin. This is mainly due to the unstable pressure wave generated by the interaction between the upstream shear layer and the downstream boundary layer. The unstable pressure waves mainly focus on the downstream zone and spread from downstream to upstream leading edge along the wall. Then, the waves interact with the shear layer so that the waves can separate from the wall to form acoustic feedback. Inside the cavity, the main unstable area concentrates on the downstream area, which leads to the result that the noise pressure level in the downstream is obviously higher than upstream.

Rossiter proposed an empirical formula for calculating the modal frequency of cavity:

$$
f_{n}=\frac{U_{\infty}}{L} \frac{n-\gamma}{M_{\infty}+(1 / \kappa)} .
$$

In the calculation of the aerodynamic noise, the most important thing is to forecast the dominant frequency location and its corresponding highest sound pressure level. Simulating results with the experimental results of the two parameters are compared in this paper. Table 1 shows the comparison of the highest sound pressure level between the numerical simulations and experiments at three locations which, respectively, are front, middle, and rear parts of the cavity bottom. Table 2 shows the comparison among the domain frequencies.

By comparison of the highest sound pressure level and the domain frequency location between NLAS simulation and experiments under different Mach numbers, it can be found that the NLAS method can simulate noise domain frequency of the cavity and the corresponding highest sound pressure level under the conditions of subsonic, transonic, and supersonic speed effectively. Some experimental values are inconsistent with values obtained from Rossiter's formula; this is because of the unsteady characteristics of the cavity flow. Rossiter's formula has its limitations, and this is why we should develop the CAA method. In the following study, we will continue to optimize the model of balance precision and computing time, making it real application in engineering calculation.

Figure 9 shows the comparison of the total sound pressure level between calculation and experiment at different locations on the bottom of the cavity. It can be seen that all the calculating results and noise pressure level at the 


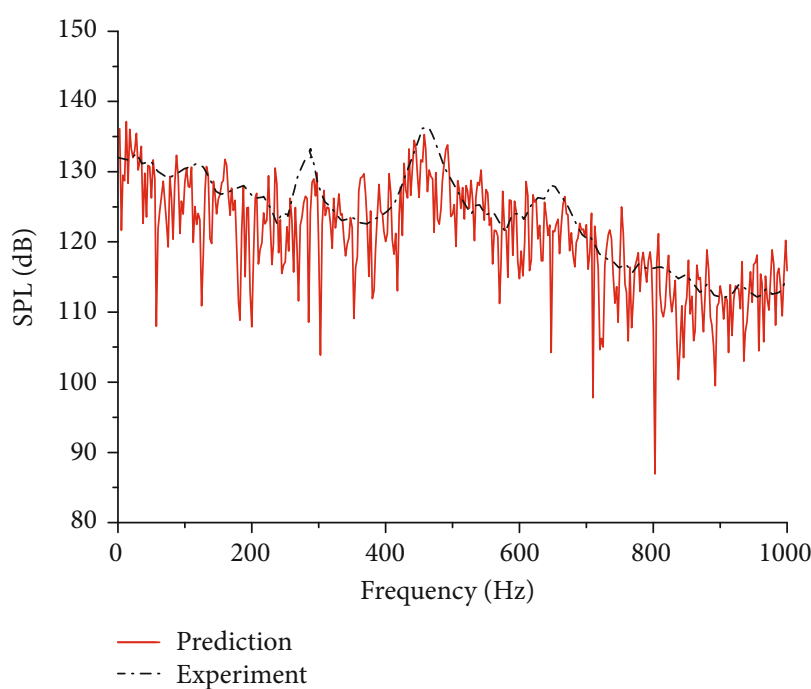

(a) $\mathrm{K} 22(X / L=0.25)$

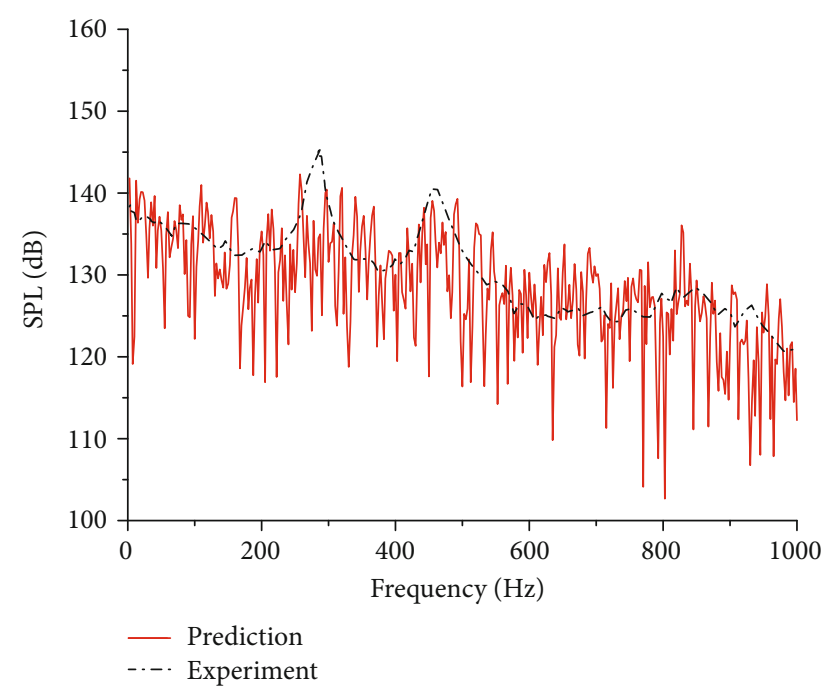

(b) $\mathrm{K} 25(X / L=0.55)$

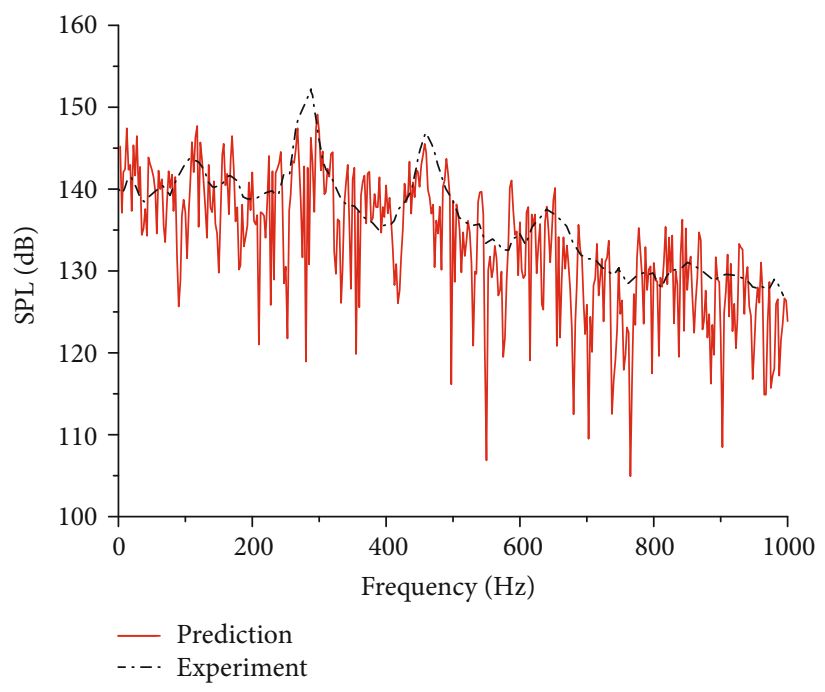

(c) $\mathrm{K} 29(X / L=0.95)$

FIgURE 6: Comparison of spectrum between calculation and test at $\mathrm{Ma}=0.6$.

testing points concentrate between $155 \mathrm{~dB}$ and $180 \mathrm{~dB}$. Besides, the sound pressure level in the upstream is a little low while that in the downstream is high. Except the place that $X / L=1$, the error between calculation and experiment on other testing points is within $5 \mathrm{~dB}$ and the experimental results are higher slightly than calculating results. This is mainly due to the existence of background noise in the wind tunnel test. The highest sound pressure level in the bottom of the cavity is over $170 \mathrm{~dB}$, so the noise inside the cavity should be controlled.

\section{Passive Control}

4.1. Numerical Model. In this paper, rectangular fence (RF) and square tooth spoiler (STS) are studied under Mach number 0.85 , and the acoustic meshes are shown as Figures 10 and 11. For rectangular fence (RF), the height is $18 \mathrm{~mm}$ and the width is $5.4 \mathrm{~mm}$. For square tooth spoiler (STS), each spoiler has a $18 \mathrm{~mm}$ height, $5.4 \mathrm{~mm}$ width, and $7.2 \mathrm{~mm}$ length.

4.2. Flow Analysis. This paper investigates the effect on noise suppression by using RF and STS passive control measures for cavity. The principle of this two passive control measures is generating disturbance in the shear layer to destroy the oscillation mechanism where it is the source of acoustic generation. Figure 12 shows oscillating pressure of a clean cavity and cavity with RF and STS from $0.2 \mathrm{~s} \sim 0.25 \mathrm{~s}$ at $X / L=0.95$ location. The adoption of passive control measures can obviously decrease the amplitude of oscillating pressure. In addition, the amplitude of oscillating pressure of STS is slightly below RF.

OASPLs for different cases are plotted in Figure 13. It is shown that the calculated OASPL agree well with the experimental result. Computational results of cavity with RF and STS imply that passive measures can lessen the total noise, 


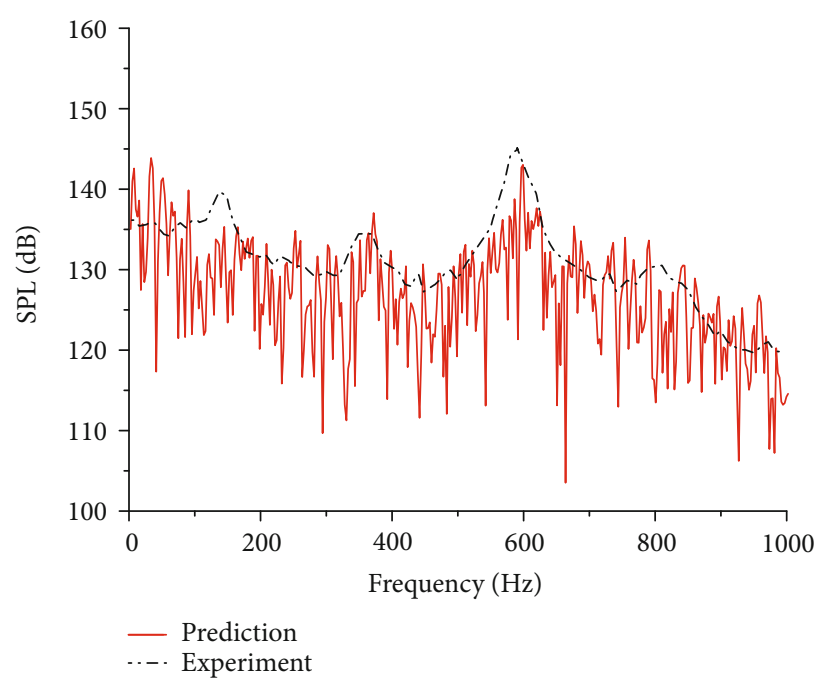

(a) $\mathrm{K} 22(X / L=0.25)$

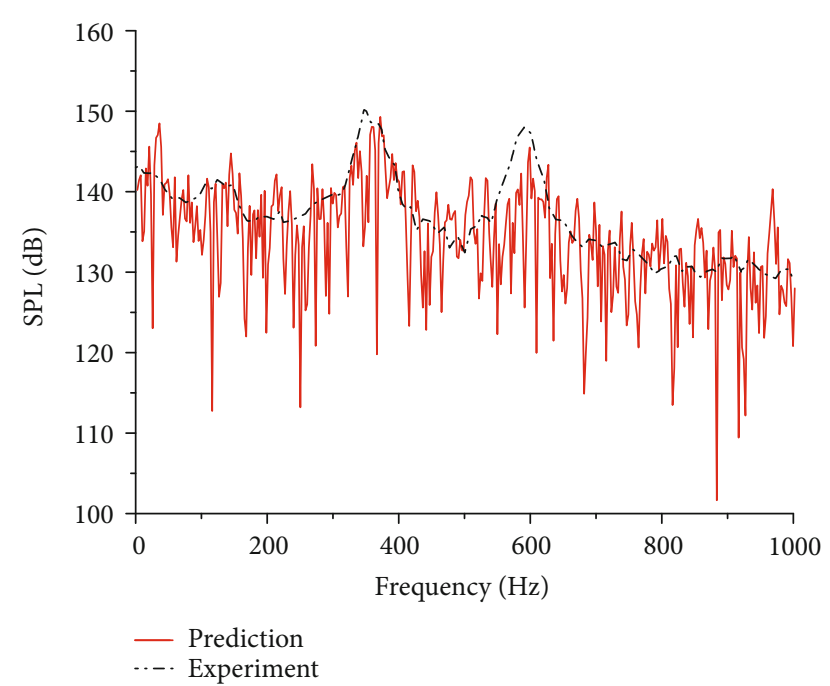

(b) $\mathrm{K} 25(X / L=0.55)$

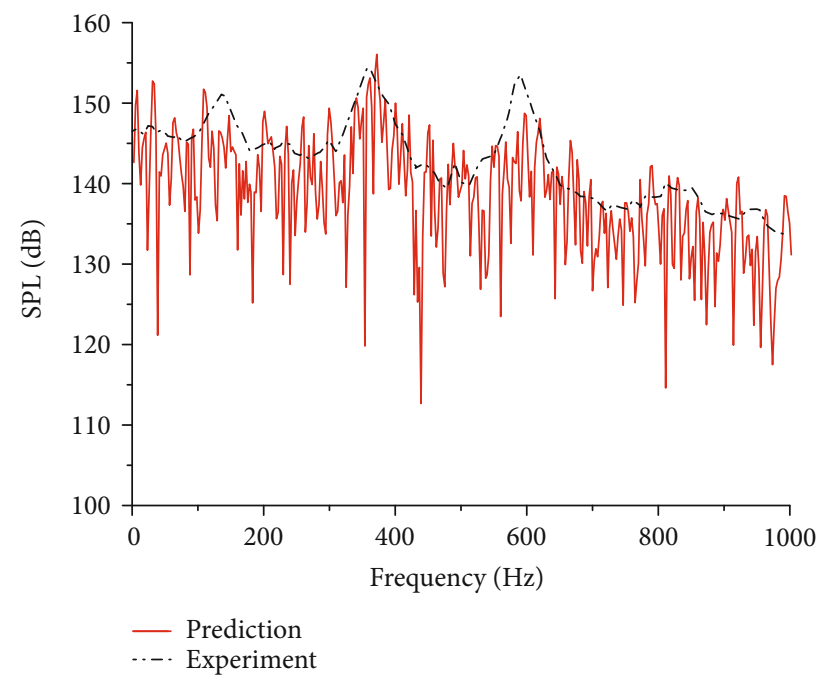

(c) $\mathrm{K} 29(X / L=0.95)$

Figure 7: Comparison of spectrum between calculation and test at $\mathrm{Ma}=0.85$.

particularly with maximum decrease of about $8 \mathrm{~dB}$ near the rear wall. It can be seen that noise levels of the cavity with RF are higher than the cavity with STS by approximately $2 \mathrm{~dB}$ on average. So, STS is more efficient to reduce flow disturbances.

Figure 14 shows sound pressure frequency spectra for 4 different situations: experiment, no control, RF, and STS. Cases (a) to (c), respectively, represent sound pressure frequency spectra at $X / L=0.25,0.55$, and 0.95 which are located at front, middle, and rear parts of the cavity. The four curves have the similar trend. When passive control measures are used, frequency spectra reduce in different degrees. Among them, STS has the better effect.

The widely used criteria for identifying the vortex named as $Q$ criteria was proposed by Hunt et al. in 1988 [31]. According to Hunt et al.'s theory, the fluid deformation can be represented by velocity gradient tensor (strain rate tensor); the tensor can be decomposed to the symmetric part of the strain rate tensor $S$ and the antisymmetric part of the tensor. If the contribution of the vortex tensor to the fluid deformation is greater than the strain rate tensor, then the existence of vortex in the region is confirmed. The $Q$ criteria is as follows:

$$
Q=\frac{1}{2}\left(\Omega_{i j} \Omega_{i j}-S_{i j} S_{i j}\right)=-\frac{1}{2} \frac{\partial u_{i}}{\partial x_{j}} \frac{\partial u_{j}}{\partial x_{i}}>0 .
$$

In the formula, the second invariant of the velocity gradient tensor is obtained from the spatial derivation of the velocity $u$; the value of the second invariant is irrelevant to the selection of the coordinate system because of the generalized invariance of the velocity gradient tensor. $Q>0$ indicates the existence of vortex, and the region surrounded by the isosurfaces defines a vortex core; conversely, there is no vortex core.

As shown in Figure 15, vortex-scale in the flow field cavity with RF and STS is obviously bigger than the clean cavity. 


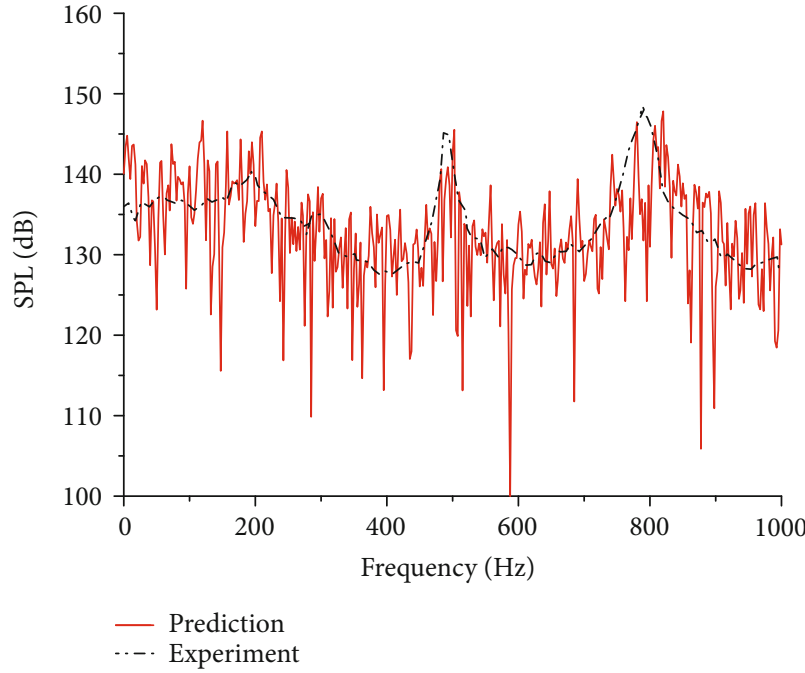

(a) $\mathrm{K} 22(X / L=0.25)$

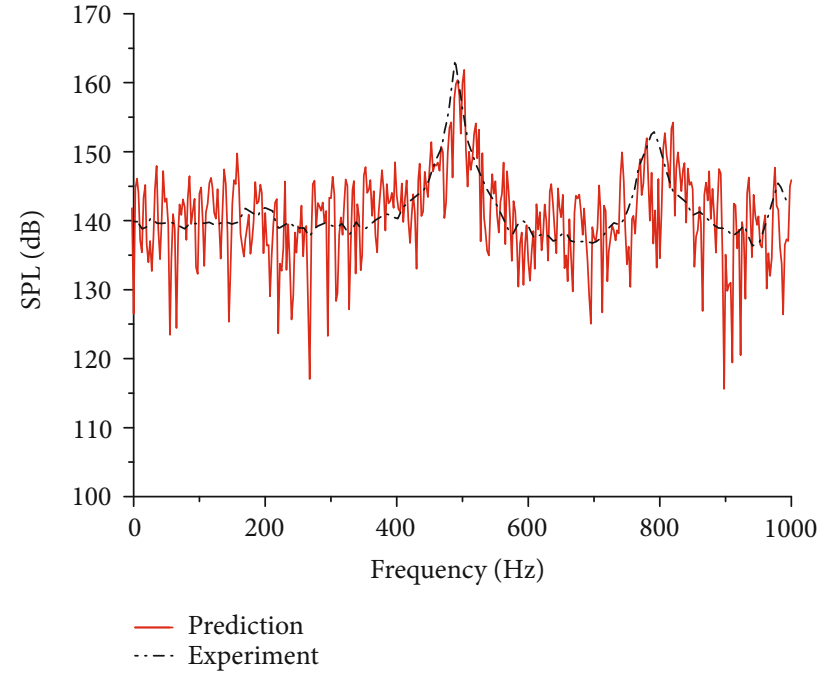

(b) $\mathrm{K} 25(X / L=0.55)$

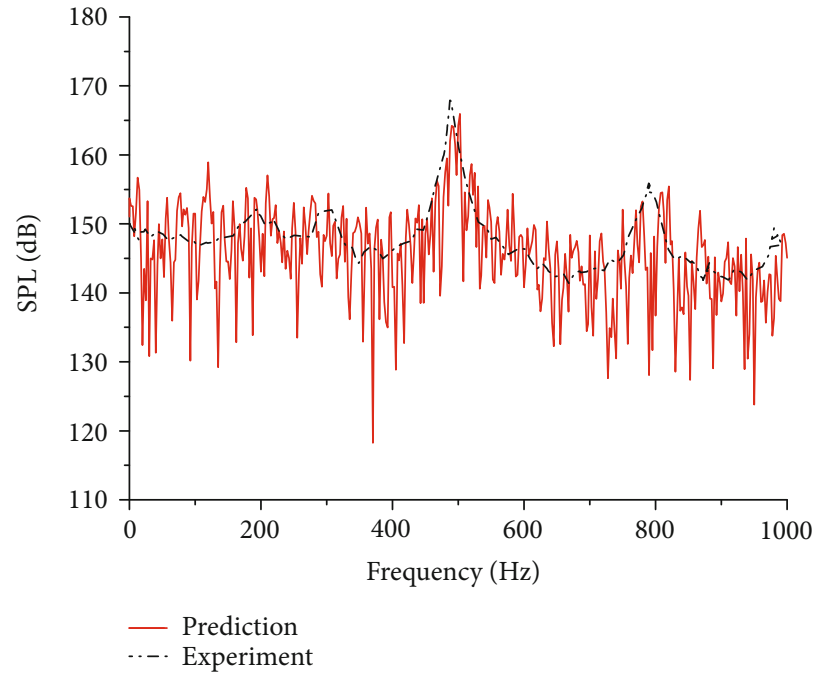

(c) $\mathrm{K} 29(X / L=0.95)$

FIgURE 8: Comparison of spectrum between calculation and test at $\mathrm{Ma}=1.35$.

TABLE 1: Comparison of the highest sound level (dB).

\begin{tabular}{lccc}
\hline Mach number & Position & Experimental data & NLAS \\
\hline \multirow{3}{*}{$\mathrm{Ma}=0.6$} & $X / L=0.25$ & 137.4 & 135.2 \\
& $X / L=0.55$ & 145.7 & 142.3 \\
& $X / L=0.95$ & 152.3 & 149.1 \\
\hline \multirow{3}{*}{$\mathrm{Ma}=0.85$} & $X / L=0.25$ & 145.2 & 143.0 \\
& $X / L=0.55$ & 150.6 & 148.1 \\
& $X / L=0.95$ & 154.7 & 156.1 \\
\hline \multirow{3}{*}{$\mathrm{Ma}=1.35$} & $X / L=0.25$ & 148.5 & 147.8 \\
& $X / L=0.55$ & 163.3 & 161.9 \\
& $X / L=0.95$ & 168.4 & 165.9 \\
\hline
\end{tabular}

Due to the existence of RF and STS spoilers, the shear layer over the cavity is shifted up away from the cavity. The incoming flow directly crosses the cavity instead of going inside of it. This phenomenon significantly reduces the intensity of flow impact on the rear wall of the cavity generating less acoustic waves. The results suggest that RF and STS could act as a vortex generator in the cavity flow field leading the vortex away from the cavity. It also can be noticed that the vortex generated by STS is broke down into small scales because of the shape of the spoiler. The small scales of the vortex could further reduce the intensity of impingement inside the cavity, which could be the reason why the STS performs better than RF on noise suppression.

\section{Conclusions}

NLAS has been presented for the prediction of M219 cavity noise with an aspect ratio of $L: D: W=5: 1: 1$ under the Mach numbers of $0.6,0.85$, and 1.35 . This method combines 
TABLE 2: Comparison of dominant frequency locations $(\mathrm{Hz})$.

\begin{tabular}{lcccc}
\hline $\begin{array}{l}\text { Mach } \\
\text { number }\end{array}$ & Position & $\begin{array}{c}\text { Experiment } \\
\text { data }\end{array}$ & NLAS & $\begin{array}{c}\text { Rossiter's } \\
\text { formula }\end{array}$ \\
\hline \multirow{M}{*}{$=0.6$} & $X / L=0.25$ & 457.5 & 459.3 & 463.8 \\
& $X / L=0.55$ & 287.6 & 257.5 & 230.8 \\
& $X / L=0.95$ & 288.2 & 297.5 & 292.5 \\
\hline \multirow{2}{*}{$=0.85$} & $X / L=0.25$ & 589.6 & 599.3 & 593.73 \\
& $X / L=0.55$ & 348.1 & 359.1 & 255.3 \\
& $X / L=0.95$ & 358.9 & 372.0 & 371 \\
\hline \multirow{3}{*}{$=1.35$} & $X / L=0.25$ & 788.7 & 820.1 & 790.9 \\
& $X / L=0.55$ & 488.8 & 502.6 & 376.25 \\
& $X / L=0.95$ & 487.7 & 502.6 & 522.8 \\
\hline
\end{tabular}

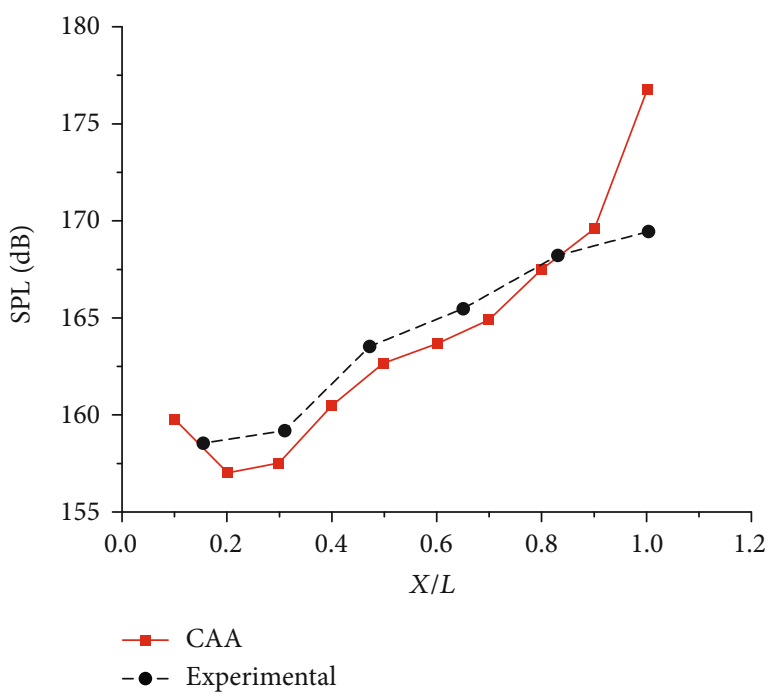

Figure 9: Comparison of OASPL distribution between CAA.

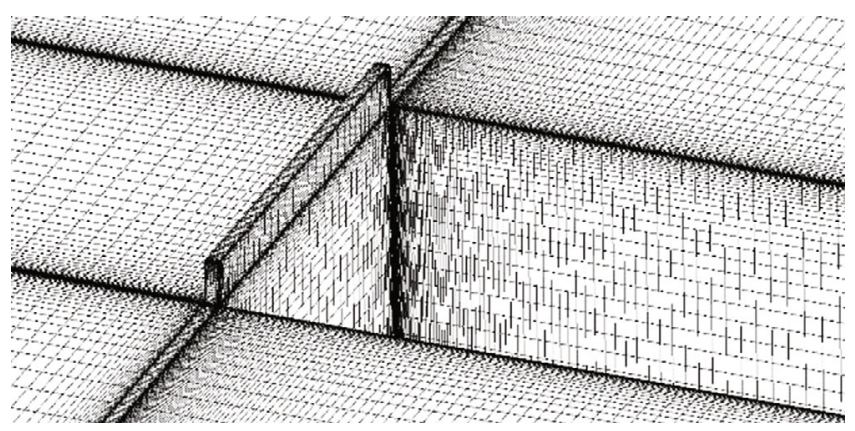

FIgURE 10: Cavity with rectangular fence.

the advantages of conventional acoustic methods and hybrid RANS/LES methods. Local Reynolds stress tensor and heat flux are obtained by calculating RANS equations on the cubic $k-\varepsilon$ model and interpolated into acoustic mesh. Then, turbulence is reconstructed based on the statistic average result, and the propagation of the pressure disturbance is solved. Reliability of the NLAS method on predicting the cavity noise is validated by comparing simulation results with experimen-

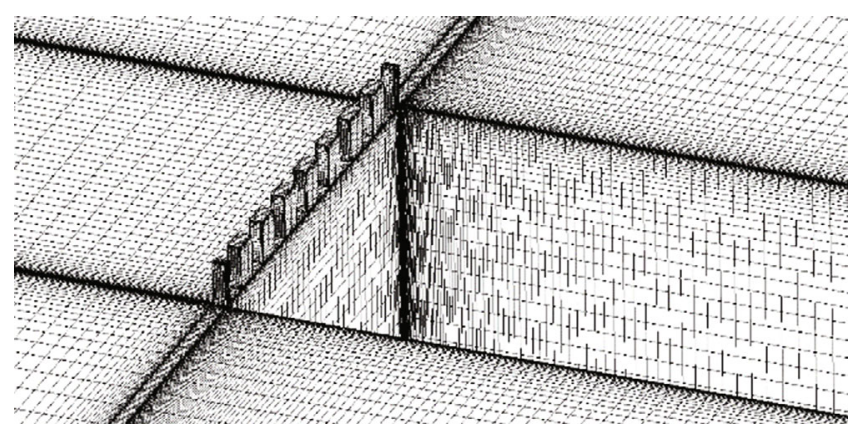

FIGURE 11: Cavity with tooth spoiler.

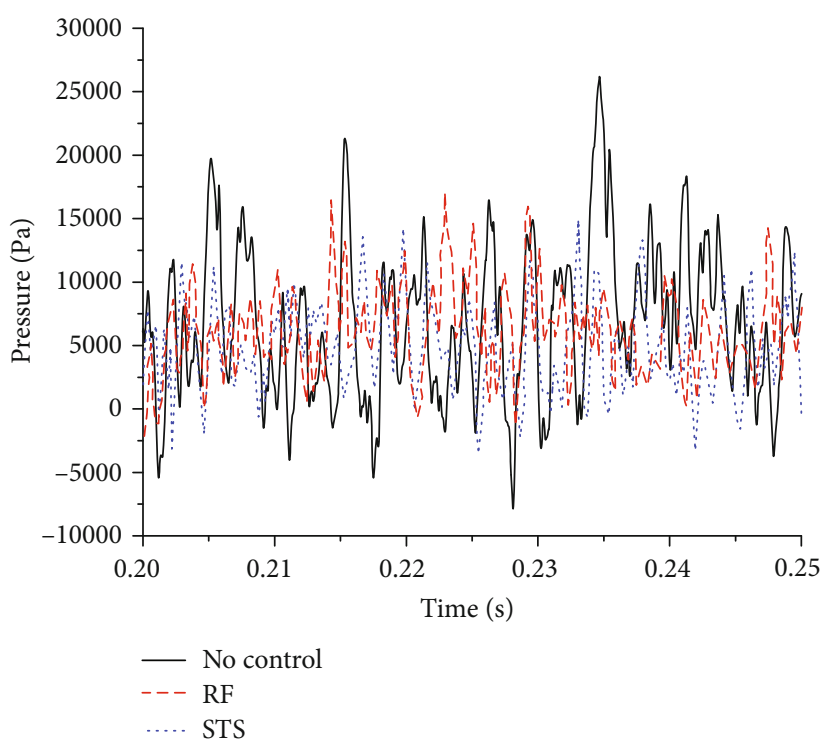

Figure 12: Pressure trace from $0.2 \mathrm{~s}$ to $0.25 \mathrm{~s}(X / L=0.95)$.

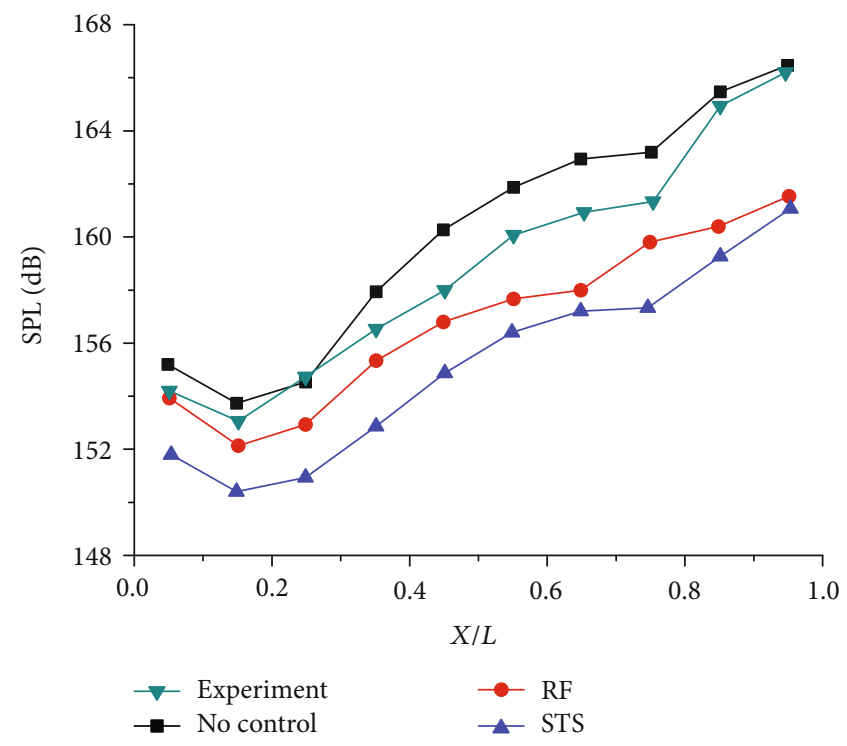

Figure 13: OASPLs for different cases. 


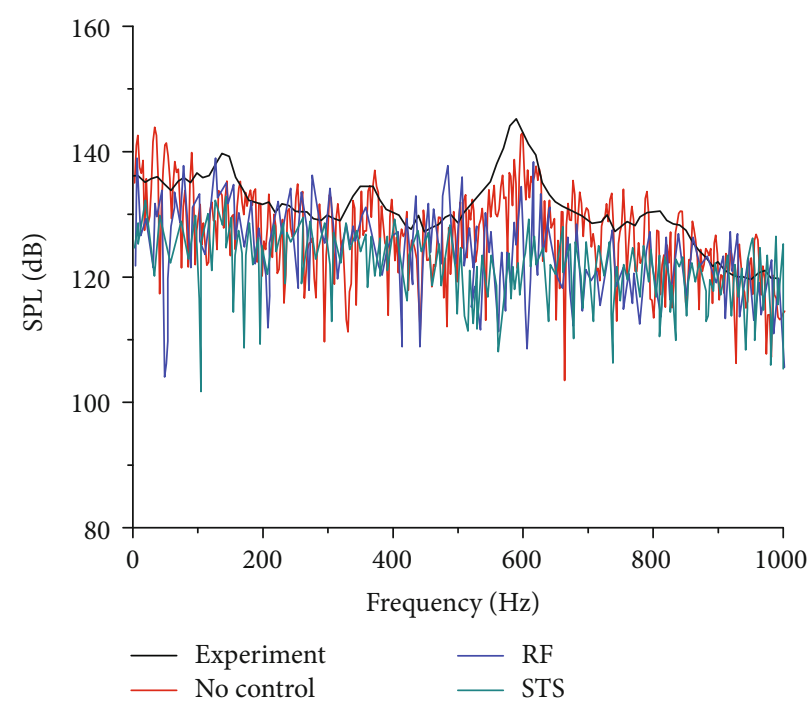

(a) $X / L=0.25$

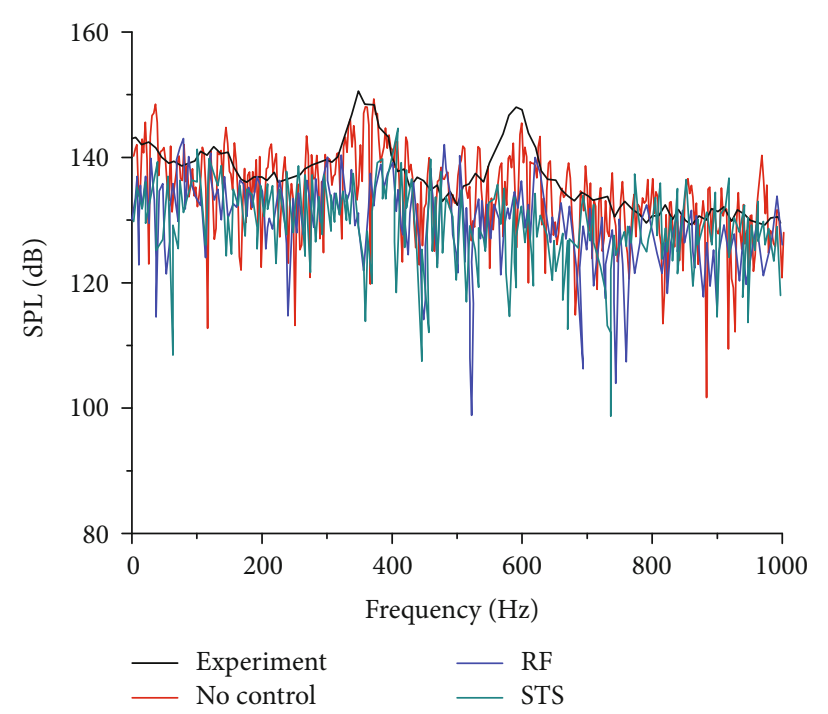

(b) $X / L=0.55$

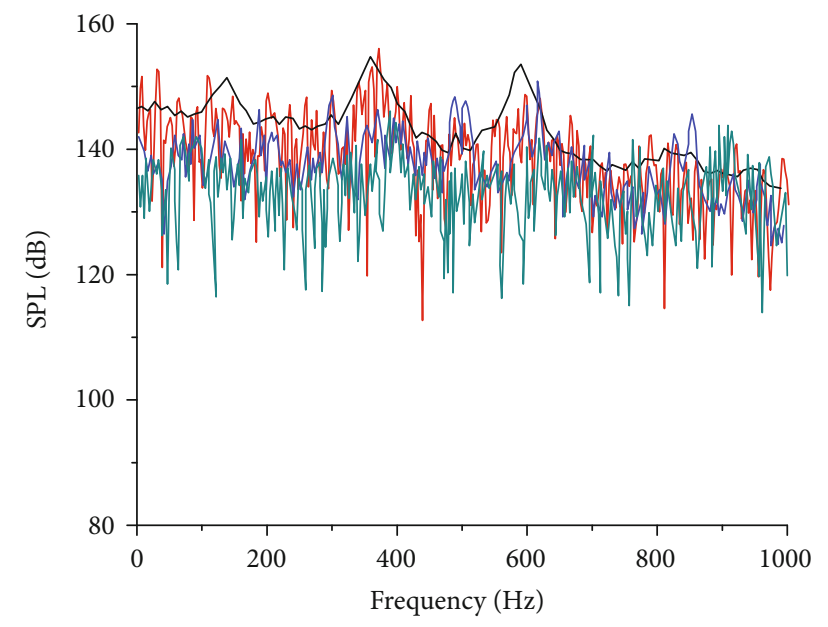

(c) $X / L=0.95$

Figure 14: Sound pressure frequency spectra.

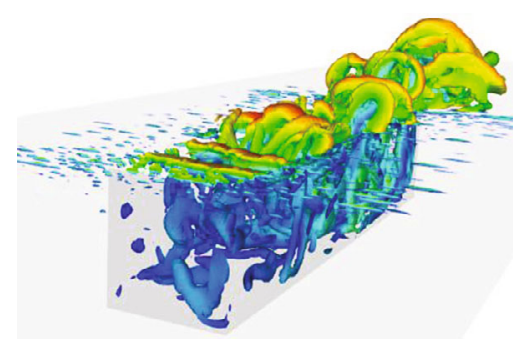

(a) Cavity without passive control

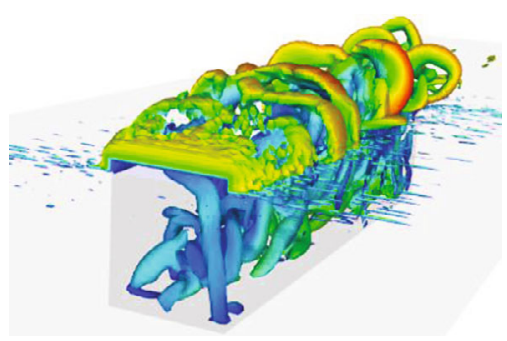

(b) Cavity with RF control

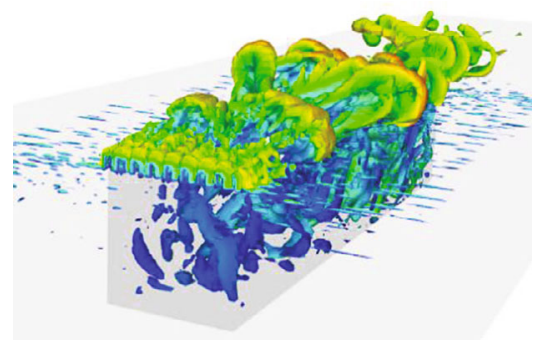

(c) Cavity with STS control

Figure 15: Isosurface of $Q$ near the cavity $\left(Q=7 \times 10^{6}\right)$.

tal results. It can be noted that NLAS has less requirements for near-wall grids and smaller computational domain than RANS, while assuring adequate precision.
Calculations with two different passive control methods are conducted under the Mach number 0.85. Computational results of the cavity with RF and STS imply that passive 
measures can lessen the total noise, and STS is more efficient. The inclusion of the fences in the experiment and comparisons with NLAS results will be researched in the future.

\section{Data Availability}

The data used to support the findings of this study are available from the corresponding author upon request.

\section{Conflicts of Interest}

The authors declare that they have no conflicts of interest.

\section{Acknowledgments}

This work is supported by the Science and Technology Research Project of Chengdu (Grant No. 2016-HM0100118-SF) and the Fundamental Research Funds for the Central Universities (Grant No. ZYGX2016J159). The experimental data in the figures and table supporting the findings of this study are from previously reported studies and datasets, which have been cited.

\section{References}

[1] X. Gloerfelt, C. Bailly, and D. Juve, "Direct computation of the noise radiated by a subsonic cavity flow and application of integral methods," Journal of Sound and Vibration, vol. 266, no. 1, pp. 119-146, 2003.

[2] O. Inoue and N. Hatakeyama, "Sound generation by a twodimensional circular cylinder in a uniform flow," Journal of Fluid Mechanics, vol. 471, pp. 285-314, 2002.

[3] J. B. Freund, S. K. Lele, and P. Moin, "Numerical simulation of a Mach 1.92 turbulent jet and its sound field," AIAA Journal, vol. 38, no. 11, pp. 2023-2031, 2000.

[4] C.-J. Tam, P. D. Orkwis, and P. J. Disimile, "Algebraic turbulence model simulations of supersonic open-cavity flow physics," AIAA Journal, vol. 34, no. 11, pp. 2255-2260, 1996.

[5] S. H. Shih, A. Hamed, and J. Yeuan, "Unsteady supersonic cavity flow simulations using coupled k-epsilon and NavierStokes equations," AIAA Journal, vol. 32, no. 10, pp. 20152021, 1994.

[6] S. Srinivasan and O. Baysal, "Navier-Stokes calculations of transonic flows past cavities," Journal of Fluids Engineering, vol. 113, no. 3, pp. 368-376, 1991.

[7] J. S. Cox, K. S. Brentner, and C. L. Rumsey, "Computation of vortex shedding and radiated sound for a circular cylinder: subcritical to transcritical Reynolds numbers," Theoretical and Computational Fluid Dynamics, vol. 12, no. 4, pp. 233253, 1998.

[8] X. Gloerfelt, F. Pérot, C. Bailly, and D. Juvé, "Flow-induced cylinder noise formulated as a diffraction problem for low Mach numbers," Journal of Sound and Vibration, vol. 287, no. 1-2, pp. 129-151, 2005.

[9] C. Cheong, P. Joseph, Y. Park, and S. Lee, "Computation of aeolian tone from a circular cylinder using source models," Applied Acoustics, vol. 69, no. 2, pp. 110-126, 2008.

[10] J. H. Seo and Y. J. Moon, "Aerodynamic noise prediction for long-span bodies," Journal of Sound and Vibration, vol. 306, no. 3-5, pp. 564-579, 2007.
[11] M. Terracol and V. Kopiev, "Numerical investigation of the turbulent flow around a truncated cylinder: noise reduction aspects," in 14th AIAA/CEAS Aeroacoustics Conference (29th AIAA Aeroacoustics Conference), p. 11, Vancouver, British Columbia Canada, May 2008.

[12] P. R. Spalart, W.-H. Jou, M. Strelets, and S. R. Allmaras, "Comments on the feasibility of LES for wings and on a hybrid RANS/LES approach," in Advances in DNS/LES: Proceedings of the First AFOSR International Conference on DNS/LES, Greyden Press, Columbus, 1997.

[13] S. T. Shih and P. J. Morris, "Parallel computational aeroacoustic simulation of turbulent subsonic cavity flows," in 6th Aeroacoustics Conference and Exhibit, vol. 32, pp. 2015-2021, Lahaina, HI, U.S.A, June 2000.

[14] A. Hamed, D. Basu, and K. Das, "Detached eddy simulation of supersonic flow over cavity," in 41st Aerospace Sciences Meeting and Exhibit, Reno, Nevada, January 2003.

[15] R. Ashworth, "Prediction of acoustic resonance phenomena for cavities using detached eddy simulation," Paper presented at the Conference of Royal Aero, 2004Society, QinetiQ, UK, 2004.

[16] Y. Liu and M. B. Tong, "Aeroacoustic investigation of a cavity with and without doors by delayed detached eddy simulation," International Journal of Aeronautical and Space Sciences, vol. 16, no. 1, pp. 19-27, 2015.

[17] P. Batten, E. Ribaldone, M. Casella, and S. Chakravarthy, "Towards a generalized non-linear acoustics solver," in 10th AIAA/CEAS Aeroacoustics Conference, Manchester, GREAT BRITAIN, May 2004.

[18] P. Batten, U. Golberg, and S. Chakravarthy, "Interfacing statistical turbulence closures with large-eddy simulation," AIAA Journal, vol. 42, no. 3, pp. 485-492, 2004.

[19] P. Batten, U. Golberg, and S. Chakravarthy, "Reconstructed sub-grid methods for acoustics predictions at all Reynolds numbers," in 8th AIAA/CEAS Aeroacoustics Conference \& Exhibit 17, Breckenridge, Colorado, June 2002.

[20] C. R. I. Silva, O. Almeida, and P. Batten, "Investigation of an axi-symmetric subsonic turbulent jet using computational aeroacoustics tools," in 13th AIAA/CEAS Aeroacoustics Conference (28th AIAA Aeroacoustics Conference), Rome, Italy, May 2007.

[21] O. Almeida and C. R. I. Silva, "Validation of radiated sound from a Mach 0,75 jet," in 19th International Congress of Mechanical Engineering, Brasilia, DF, November 2007.

[22] J. Ross and J. Peto, "The effect of cavity shaping, front spoilers and ceiling bleed on loads acting on stores, and on the unsteady environment within weapon bays," QinetiQ Report, Portsmouth, UK, 1997.

[23] L. N. Cattafesta, D. Williams, C. Rowley, and F. Alvi, "Review of active control of flow-induced cavity resonance," in 33rd AIAA Fluid Dynamics Conference and Exhibit, Orlando, Florida, June 2003.

[24] J. Rossiter, "Wind tunnel experiments on the flow over rectangular cavities at subsonic and transonic speeds," Ministry of Aviation; Royal Aircraft Establishment; RAE Farnborough, Farnborough, UK, 1964.

[25] J. Ross and J. Peto, Internal Stores Carriage Research at RAE, Royal Aircraft Establishment TR 2233, Bedford, England, U.K., 1992.

[26] J. Ross and J. Peto, "The effect of cavity shaping, front spoilers and ceiling bleed on loads acting on stores, and on the 
unsteady environment within weapon bays," Royal Aircraft Establishment TR 2233, Bedford, England, U.K., 1997.

[27] L. Shaw, R. Clark, and D. Talmadge, "F-111 generic weapons bay acoustic environment," Journal of Aircraft, vol. 25, no. 2, pp. 147-153, 1988.

[28] B. Smith, T. Welterlen, B. Maines, L. Shaw, M. Stanek, and L. Grove, "Weapons bay acoustic suppression from rod spoilers," in 40th AIAA Aerospace Sciences Meeting \& Exhibit, Reno, NV, U.S.A, January 2002.

[29] L. Ukeiley, M. Ponton, J. Seiner, and B. Jansen, "Suppression of pressure loads in cavity flows," AIAA Journal, vol. 42, no. 1, pp. 70-79, 2004.

[30] M. J. C. De Henshaw, "M219 cavity case' in 'Verification and validation data for computational unsteady aerodynamics," Tech Rep RTOTR-26, AC/323(AVT)TP/19, QinetiQ, UK, 2002.

[31] J. C. R. Hunt, A. A. Wray, and P. Moin, "Eddies, stream and convergence zones in turbulent flows, report CTR-S88," Center For Turbulence Research, p. 193, 1988. 


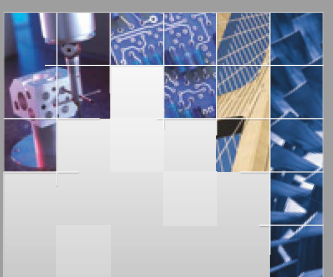

\section{Enfincering}
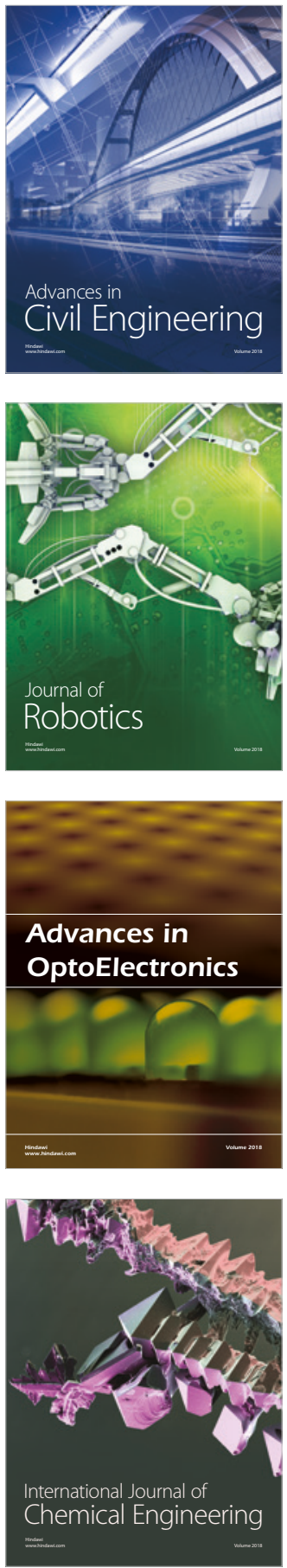

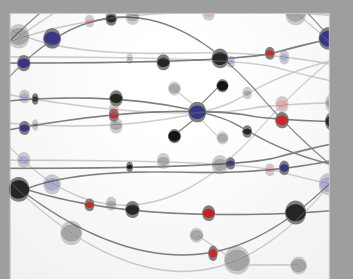

\section{Rotating \\ Machinery}

The Scientific World Journal

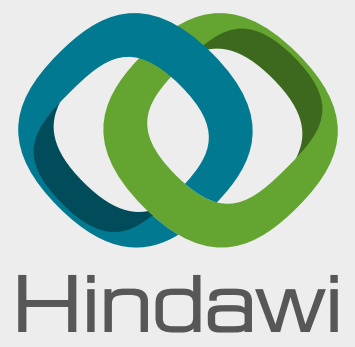

Submit your manuscripts at

www.hindawi.com
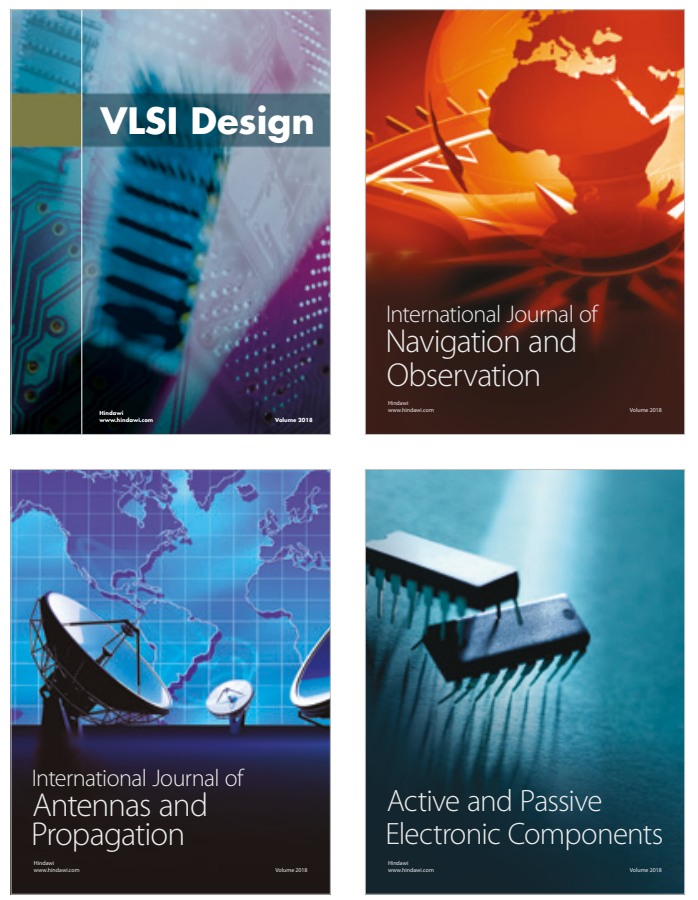
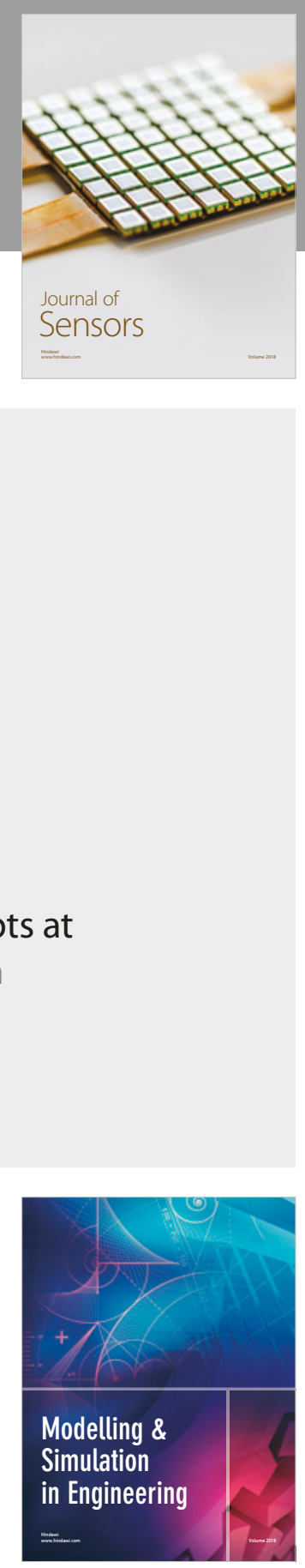

\section{Advances \\ Multimedia}
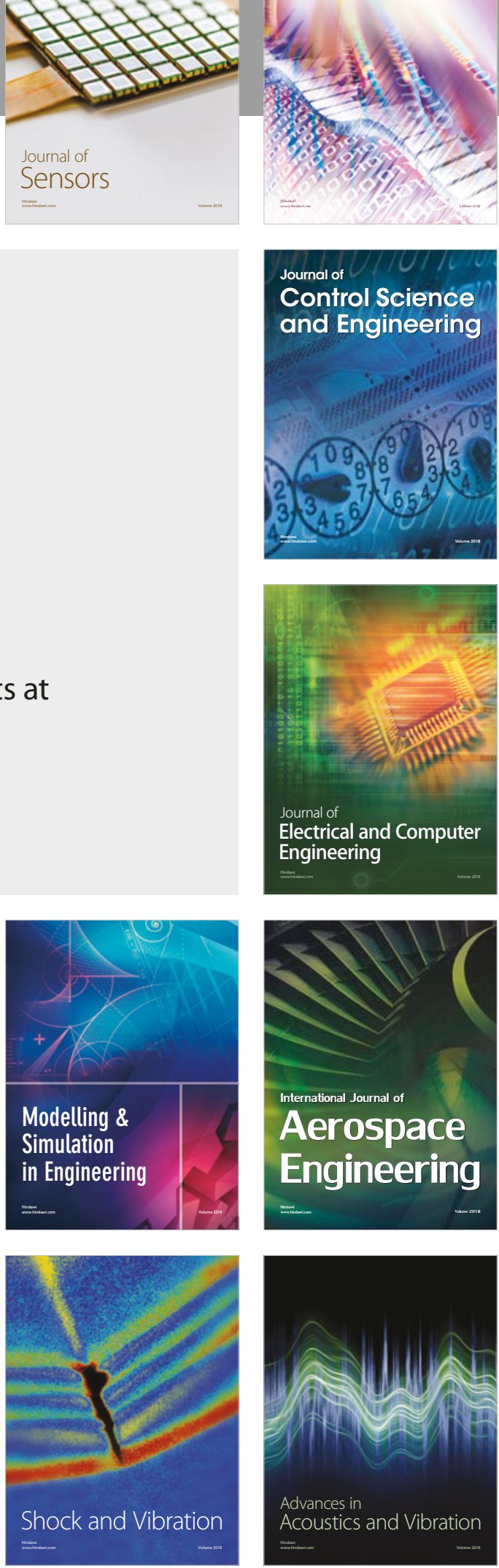